\title{
VARIA
}

\section{Pour une correction phonétique personnalisée en FLE ou de la remédiation « sur mesure »}

\section{« Custom made » error correction of pronunciation in French as a foreign language}

\author{
Magdalena Dańko \\ Université d'Opole \\ mdanko@uni.opole.pl \\ Dominique Hamm \\ Université de Strasbourg \\ dominique.hamm.ips@gmail.com
}

\begin{abstract}
Herein we would like to rise up a concern of the error correction of pronunciation in French as a Foreign Language. The results of the experimental acoustic analysis (concerning the values of acoustic vowel formants F1 and F2) enabled to precisely specify the difficulties of the experiment's participants in a field of French vocal system. In relation to the differences of vocal systems of French and Polish language we suggest custom solutions on a didactic level. Based on verbo-tonal method for phonetic integration the error correction activities had been individually elaborated for each participant.
\end{abstract}

Keywords: didactics of phonetics of the French language, French pronunciation teaching, contrastive phonetics, interlanguage, Polish-speaking learners

\section{INTRODUCTION}

La disponibilité d'outils informatiques de plus en plus performants permet d'effectuer des analyses de plus en plus fines dans le domaine de la phonétique, notamment lors d'une approche contrastive, et, y compris au niveau de la phonétique 
corrective. Une aubaine pour la didactique de l'oral du français langue étrangère (FLE) et plus précisément sa dimension phonétique, car enseigner la prononciation du français à des apprenants non natifs reste un défi. Des analyses formantiques avancées contribuent à l'établissement de valeurs de formants en français et ce à partir d'un nombre très important de réalisations d'un même phonème, avec la prise en compte des sources de variabilité bien définies à présent, telles que le contexte phonétique immédiat et la position du phonème dans le mot et dans le groupe rythmique (cf. Gendrot, Adda-Decker, 2006). En l'occurrence, des analyses acoustiques et perceptives permettent «la création d'outils intégrés de formalisation, de représentation, de remédiation et d'évaluation » selon la langue d'origine des apprenants (Landron et al., 2010, p. 73 ; consulter aussi : Kamiyama, Vaissière, 2017 ; Hamm, Dańko, 2017 ; Dańko, Sauvage, Hirsch, 2015 ; Racine et al., 2012). Les initiatives comme le Corpus PhoDiFLE (Landron et al., 2010) et I.P.F.C. (Detey, Kawaguchi, 2008; Racine et al., 2012) voient le jour et permettent d'aborder autrement l'enseignement des sons du français (système vocalique, système consonantique) et de la prosodie.

L'informatique permet l'étude systématique de très grandes quantités de réalisations d'un même phonème. Elle permet d'avoir une représentation visuelle des segments via le spectrogramme, d'effectuer des mesures semi-automatiques (avec vérification manuelle) et reproductibles, et offre la possibilité de dégager des tendances générales.

L'étude des réalisations des phonèmes ne se place donc plus dans une approche par transcription API, globalisante et subjective. Au contraire, les productions des apprenants sont situées par rapport à des données chiffrées, et des variations visualisables sur des graphiques. (Landron et al., 2010, p. 75-76)

D'une part, la notion de norme, inaccessible, évolutive, pose toujours problème en didactique du FLE, d'autre part, il n'est pas possible d'enseigner/apprendre sans avoir de références. Le français de référence constitue actuellement la norme en vigueur, il s'étend dans la moitié nord de la France. Néanmoins, norme linguistique et norme pédagogique ne sauraient être confondues : il faut garantir à l'apprenant un dénominateur commun n'épuisant pas nécessairement toutes les spécificités régionales, socio-culturelles ou individuelles, mais permettant l'intercompréhension. Nous décidons de ce fait d'étudier les écarts entre les réalisations des apprenants et les tendances générales des locuteurs natifs telles qu'elles ont été définies par les études les plus récentes à ce sujet (Georgeton et al., 2012). Le décalage constitue pour nous le point de départ d'un processus de correction. Le phénomène de crible phonologique (Troubetzkoy, 1949) reste à l'origine de ce décalage ; lorsque celui-ci est trop important et provoque des troubles empêchant la communication, les procédés de la phonétique corrective entrent naturellement en jeu. Certes, l'intercompréhension est le facteur indispensable par excellence, mais n'est pas « la seule vraie garante » 
de ce que l'on peut appeler «les bonnes prononciations» (Landron et al., 2010, p. 76). Landron et al. l'ont bien compris :

(...) en tant que didacticiens, nous devons viser la production du français natif pour nos apprenants. Même si, avec l'enjeu identitaire, certains apprenants ne veulent pas perdre leur accent d'origine - et dans un tel cas, ils ne modifieront sans doute pas leur façon de parler - d'autres veulent acquérir une façon de parler non marquée (Pillot-Loiseau et al., 2010) et le devoir de l'enseignant de FLE est de les y aider (Lauret, 2007). (Landron et al., 2010, p. 77)

À l'heure où l'on parle de glottophobie (Blanchet, 2016), un phénomène qui pourrait aussi susciter cette envie de «se débarrasser de l'accent étranger » chez les allophones, nous nous interrogeons (et c'est un travail à long terme) sur la possibilité d'acquérir une façon de parler non marquée, surtout s'il s'agit d'un enseignement dans un cadre institutionnel, dans un pays où la langue apprise est étrangère.

Dans notre précédente étude «Perception et traque de l'accent étranger : la production des voyelles orales en FLE par des polonophones », nous avons identifié et décrit les sources d'interférences chez les apprenants polonais participant à cette étude expérimentale. Dans ce texte, nous allons tenter de proposer une remédiation aux difficultés réellement rencontrées, en reprenant comme point de départ les résultats de l'analyse acoustique effectuée (Hamm, Dańko, 2017). Ainsi, les propos qui vont suivre s'inscriront dans le continuum de l'étude acoustique contrastive des systèmes phonologiques du polonais et du français déjà réalisée, citée ci-dessus.

\section{DOMAINE DE RECHERCHE, MÉTHODOLOGIE, OBJECTIFS}

Nous rappelons que notre recherche se situe dans les domaines de la phonétique et de la didactique de l'oral du FLE. Notre arrière-plan théorique se fonde sur les modèles d'acquisition des sons en langue étrangère, notamment ceux de Polivanov (1931), Troubetzkoy (1949), Best (1994), Kuhl (1991) et Flege (1995). Notre objet d'étude est l'ensemble de réalisations phonétiques du français oral produites par quatre locutrices polonophones enregistrées dans le cadre d'une étude acoustique expérimentale à visée essentiellement didactique (Hamm, Dańko, 2017). Or, nous avons construit deux corpus (un français et un polonais), entièrement contrôlés, selon une démarche expérimentale, focalisés sur les points précis à analyser. Premièrement, le corpus polonais avait pour but d'établir un espace vocalique (visualisation triangulaire des deux premiers formants, F1 et F2, pouvant également être interprétée en termes articulatoires) pour chaque locutrice, de 6 voyelles de base en langue maternelle. Il se compose d'énoncés porteurs de mots phonétiques comprenant les voyelles étudiées (fragments de poèmes, comptines, proverbes et d'autres énoncés déclaratifs, interrogatifs ou exclamatifs). Au total 73 voyelles ont été 
mesurées pour le corpus polonais (F1 et F2) de chaque locutrice. Deuxièmement, l'objectif du corpus français, quant à lui, était d'établir un espace vocalique de 10 voyelles de base en langue cible pour chaque locutrice. Des énoncés porteurs de mots phonétiques, comprenant les voyelles étudiées, se composent de vers prélevés dans des textes au programme de Première L des lycées, ainsi que d'autres énoncés simples déclaratifs, interrogatifs, exclamatifs ou injonctifs. Les connaissances phonologique, prosodique, lexicale et morphosyntaxique d'un apprenant de niveau intermédiaire (B1) ont toujours été prises en compte. Au total 117 voyelles (F1 et F2) ont été mesurées pour le corpus français de chaque locutrice, et ce dans le contexte le plus adéquat: généralement sous l'accent rythmique hypothétique (fin de mot phonétique). Les enregistrements sonores des corpus ont été réalisés au mois de novembre 2015, dans le studio insonorisé de l'Institut de Phonétique de Strasbourg (IPS, Université de Strasbourg), à l'aide d'un enregistreur numérique Fortex Stéréo FR2 sur carte Compact Flash (réglage de qualité : 44,1 kHz/16 bits) et à un microphone directionnel Sennheiser e835S. Le son a été analysé au moyen du logiciel d'analyse acoustique Praat (Boersma, Weenick, 2010) qui permet de visualiser le spectre acoustique et de relever les valeurs des deux premiers formants (F1 et F2) responsables du timbre des voyelles concernées par l'étude. Les mesures, toutes manuelles, ont été prises au milieu de la structure formantique stable des voyelles et vérifiées à l'aide de l'onglet «Formant listing ». Finalement, les espaces vocaliques de chaque locutrice, définis grâce aux données des deux corpus (français et polonais), ont été superposés afin d'observer les différences de localisation des voyelles dans cet espace commun.

Nous souhaitons à présent: 1) lister des écarts phonétiques produits par ces quatre locutrices par rapport aux valeurs de références de Georgeton et al. (2012, p. 2) afin de trouver une réponse didactique adaptée aux interférences à dépasser, pointées en détail dans l'étude précédente (Hamm, Dańko, 2017), visualisées de manière synthétique sur les graphiques (figures 1-4 ci-dessous). Pour y parvenir, nous privilégions la méthode verbo-tonale de correction phonétique (Guberina, 1971 ; Intravaia, 2000 ; Billerey, 2004 ; Billières, 2005), car les premiers résultats auprès du public polonophone nous ont confirmé son efficacité (Dańko, Sauvage, Hirsch, 2015, 2017) et offrent des perspectives intéressantes.

En ce qui concerne nos données de référence au niveau de l'espace vocalique polonais, nous avons repris les valeurs formantiques des voyelles polonaises de Wierzchowska $(1965,1980)$. Quant à l'espace vocalique français, visualisé chaque fois en comparaison avec les résultats de nos quatre locutrices (figures 1-4 ci-dessous), il a été conçu à partir de l'étude de Georgeton et al. (2012). Or, il est important de noter que Georgeton et al. ont défini les caractéristiques acoustiques des voyelles françaises en contexte isolé, même si ces voyelles isolées ont été chaque fois extraites d'une phrase cadre (nous citons un exemple: «Bébé, il a dit 'é' comme dans bébé », Georgeton et al., 2012, p. 146). Ceci n'est pas le cas de nos corpus qui 
comprennent des voyelles prononcées dans un cadre mêlant les contextes consonantiques et prosodiques. D'ailleurs, en général, notre but n'est pas d'aligner nos étudiants à une norme, mais de les rapprocher des tendances du français parlé des natifs.

\section{CADRE COMMUN DES PROCÉDÉS DE REMÉDIATION PROPOSÉS}

La remédiation proposée sera adaptée aux difficultés rencontrées. Elle prendra en compte les apports de la méthode verbo-tonale d'intégration phonétique (MVT) et de la phonétique combinatoire (cf. Intravaia, 2000 ; Callamand, 1981) dont nous exploitons les principes, tels que :

- l'aspect prosodique, l'intonation montante ou descendante, afin de travailler le caractère tendu ou relâché, clair ou sombre d'un son (la MVT utilise l'intonation montante afin d'accentuer d'abord le caractère clair des voyelles et la tension des consonnes; l'intonation descendante, quant à elle, accentue d'abord le caractère sombre des voyelles et le relâchement des consonnes) ;

- l'entourage consonantique facilitant également le caractère tendu/relâché, clair/sombre du son qui pose problème.

La position accentuée sera privilégiée malgré tout, même pour relâcher ou assombrir un phonème : nous placerons chaque fois le son problématique au sein de la dernière syllabe du groupe rythmique puisque c'est la position la plus importante quant à la perception/compréhension.

\subsection{OPPOSITION DES TRAITS : CARACTÈRE CLAIR/SOMBRE, CARACTÈRE TENDU/RELÂCHÉ DU SON EN QUESTION}

Pour des raisons didactiques, à ce stade, et en raison d'une remédiation dans la perspective verbo-tonale, les procédés de remédiation se limiteront à un nombre restreint de traits :

- l'opposition des traits aigu/grave (les composantes claires/sombres);

- l'opposition des traits de tension/relâchement.

En fait, l'apparition d'interférences découle, entre autres, d'une mauvaise appréciation des degrés d'acuité ou de tension et c'est sur ces degrés que nous allons nous focaliser ci-dessous.

\subsection{DIAGNOSTIC D'ERREURS}

Un diagnostic individuel d'erreurs de prononciation à partir des résultats de notre étude acoustique (Hamm, Dańko, 2017) précède la remédiation. Il s'agira de déterminer : a) la sur- ou sous-estimation du degré d'acuité ; b) la sur- ou sousestimation du degré de tension. 
En ce qui concerne le public polonophone en général, les interférences concernent notamment les voyelles orales de moyenne aperture mi-fermées, /e, $\varnothing, \mathrm{o} /$, et les deux voyelles antérieures labialisées /y/ et /œ/ du français. Pour nos quatre locutrices, il s'agit d'un problème de tension des voyelles tendues en français /e, $\mathrm{o} /$ et/ou d'une mauvaise appréciation du degré d'acuité des voyelles $/ \mathrm{y} /-/ \mathrm{u} /$ ou encore $/ œ /$.

\subsection{DU CONTEXTE FAVORISANT AU CONTEXTE DÉFAVORABLE}

En règle générale, nous proposerons de placer le son à corriger au sein d'un mot phonétique porteur, dans un cadre très favorisant - favorisant - pour arriver à un cadre défavorable, car l'apprenant devrait pouvoir fonctionner in situ dans celui-ci aussi en langue étrangère. Par le cadre favorable/défavorable, nous comprenons alors les conditions favorables/défavorables à la perception et à la production de sons (sur lesquelles influent la position dans le groupe rythmique, la courbe intonative ascendante/descendante, le sommet/creux intonatif, l'entourage consonantique ou vocalique immédiat). Il s'agirait de commencer par des logatomes, si possible, d'inclure ensuite des unités de sens avec des énoncés plus courts (de deux à trois syllabes), pour passer ensuite à des énoncés plus longs (de quatre à huit syllabes). Des logatomes nous seront utiles dans la mesure où le contexte sémantique est un facteur complexifiant le processus d'acquisition de sons nouveaux que nous souhaiterions écarter de prime abord en vue de faciliter la perception et la production. Les aspects prosodiques (l'intonation montante ou descendante) seront primordiaux dans la correction que nous souhaitons proposer selon l'optique verbo-tonale (p. ex. Intravaia, 2000), car prometteuse d'après nos études récentes auprès du public polonophone (Dańko, Sauvage, Hirsch, 2015, 2017). Comme nous l'avons déjà évoqué, nous jouerons aussi sur la variation de l'entourage consonantique qui, lui aussi, aurait son rôle facilitateur ou non. Nous avons choisi de présenter quatre cas isolés, car si les principes de la MVT peuvent être généralisés pour un groupe linguistique donné en fonction de prédictions fondées sur une étude comparative des systèmes phonologiques de la langue source et de la langue cible, il ne faut pas oublier qu'elle est un savoir-faire et un savoir-être reposant sur l'empathie, la congruence. La prise en charge de l'apprenant se doit d'être précoce et surtout individualisée : il faut lui fournir le procédé idoine par une intervention directe dans l'émission des énoncés et/ou par la préparation d'exercices correspondant à des besoins réels identifiés. Nous espérons que notre exemplification servira à d'autres enseignants, nos propositions de remédiation visent une libération du champ perceptuel de l'apprenant, mais également l'autonomie et l'auto-correction. 


\section{POUR UNE CORRECTION PERSONNALISÉE OU DE LA REMÉDIATION «SUR MESURE »}

\section{1. ÉTUDE DE CAS : LOCUTRICE 1 (B2 CECRL)}

Au moment de l'étude (2015/2016) : cette locutrice apprend le français depuis six ans, elle a commencé au lycée en Pologne. Elle a terminé ses études de licence en philologie romane à l'Université d'Opole en 2015. Après les études, elle est partie en France pour faire un stage dans une société à Strasbourg et perfectionner son français. Elle parle français (niveau B2), italien (niveau A2), tchèque (niveau A2), slovaque (niveau B1) et anglais (niveau A2). Le polonais est sa langue maternelle.

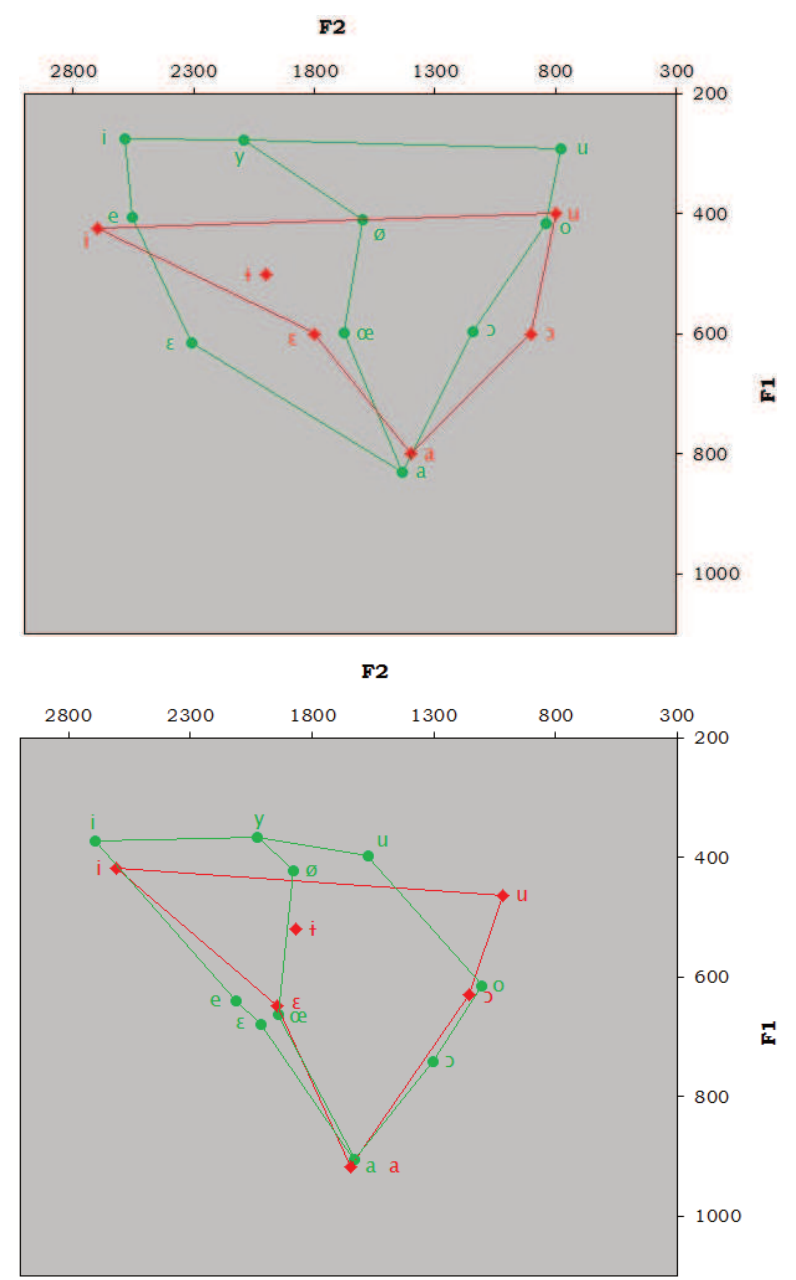

Figure 1 - Locutrice 1 : Superposition des espaces vocaliques des deux langues - valeurs formantiques

$(\mathrm{Hz})$ de référence (à gauche), valeurs moyennes formantiques $(\mathrm{Hz})$ de la locutrice 1 (à droite) 
Remédiation proposée à partir du diagnostic effectué (Hamm, Dańko, 2017, voir figure 1 pour la synthèse).

En ce qui concerne la locutrice 1 :

- la remédiation proposée viserait la distinction des voyelles /y/ et /u/ en insistant sur le caractère clair du /y/ et le caractère sombre du /u/ ;

- il s'agirait de renforcer le caractère tendu du /e/ ;

- l'objectif serait aussi d'assombrir le /œ/ ;

- ainsi que de tendre le /o/.

Méthodologie de remédiation

- Distinguer les voyelles /y/ et /u/

Nous proposerions de favoriser les composantes claires de la voyelle /y/ et d'insister sur les composantes sombres de la voyelle /u/. Les procédés de correction consisteraient alors à placer le $/ \mathrm{y} / \mathrm{en}$ fin de courbe intonative ascendante et de mettre le $/ \mathrm{u} /$ en fin de courbe intonative descendante, d'accompagner le $/ \mathrm{y} /$ de consonnes dites « éclaircissantes » $[\mathrm{s} \mathrm{z} \mathrm{t} \mathrm{d} \mathrm{n]} \mathrm{et} \mathrm{le} \mathrm{/u/} \mathrm{de} \mathrm{consonnes} \mathrm{«} \mathrm{assombrissantes} \mathrm{»}[\mathrm{p} \mathrm{b} \mathrm{m} \mathrm{f} \mathrm{v}]$. Puis, passer progressivement du contexte favorisant au contexte défavorable. Cidessous, nous présentons quelques exemples. En ce qui concerne la transcription phonétique, nous aurions pu, en syllabe inaccentuable, utiliser des archiphonèmes quant aux quelques voyelles de moyenne aperture, mais nous avons préféré opérer un choix entre voyelle ouverte ou voyelle fermée (en syllabe fermée inaccentuable, la tendance étant de toute manière à garder une voyelle ouverte et, en syllabe ouverte inaccentuable, la tendance étant à la neutralisation). Nous fournissons ainsi un modèle unique de remédiation, la position inaccentuable étant absolument moindre sur le plan linguistique quant à l'émergence du sens, rappelons-le.

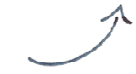

[a't y ]

[a's y ]

$\left[a^{\prime} z y\right]$

[dada't y ]

[kata'dy ]

[pata'ny]

[aba'ty]

[vwa'ty]

[ $\varepsilon$ la's y ]

[tyla'vy]

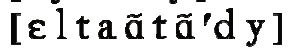

[ilta'ply]

[illaa 'te/eilla'by]

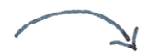

[a'f u ]

[a'p u ]

[d $a^{\prime} b u$ ]

[da'vu]

[a'm u]

[seta'vu]

[ile'f u]

[ $\varepsilon l \mathrm{e}^{\prime} \mathrm{pur}$ ]

[bada'bum]

[kel'f ul]

[s e'd u]

[s ne pa'mu]

[s e m $\tilde{\nu} \int u^{\prime} \int u$ ]

[i l a a b b̃'g u] 


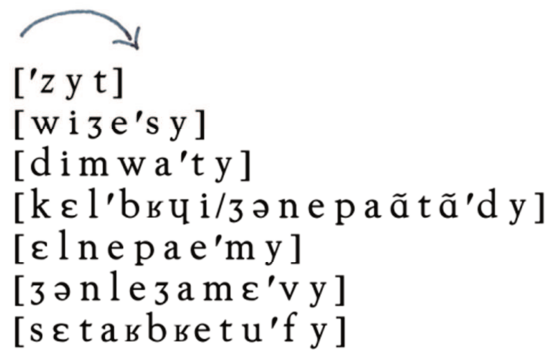

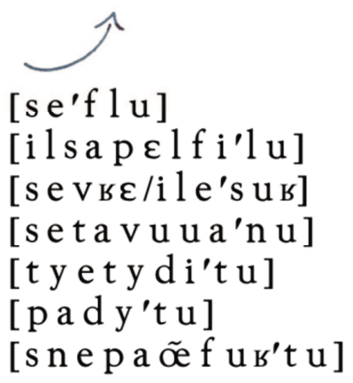

- Renforcer le caractère tendu du /e/

Pour insister sur le caractère tendu du /e/, nous conseillerons de placer la voyelle au sein de la syllabe accentuée en fin de courbe intonative ascendante, accompagnée des consonnes [p, t, s], car ce contexte d'après notre dernière étude (Dańko, Sauvage, Hirsch, 2017) constitue l'entourage le plus favorisant à l'acquisition (pour ensuite changer de contexte pour passer à celui moins favorable). Il serait question de privilégier un flux de la parole plus rapide, en incluant un geste qui accompagnerait le travail sur le rythme et l'accentuation (taper les mains, taper les pieds ou utiliser un objet pour marquer la syllabation et stimuler l'accent), parler plus rapidement a une incidence sur la tension.

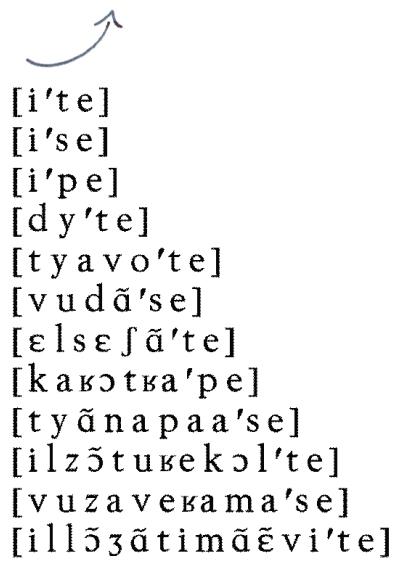

- Assombrir le /œ/

Pour assombrir le /œ/, il serait question de placer cette voyelle en fin de courbe intonative descendante, accompagnée des consonnes $[\mathrm{p} \mathrm{b} \mathrm{m} \mathrm{f} \mathrm{v}$, pour créer un contexte favorable à l'acquisition. Puis, progressivement, passer au contexte défavorable. 

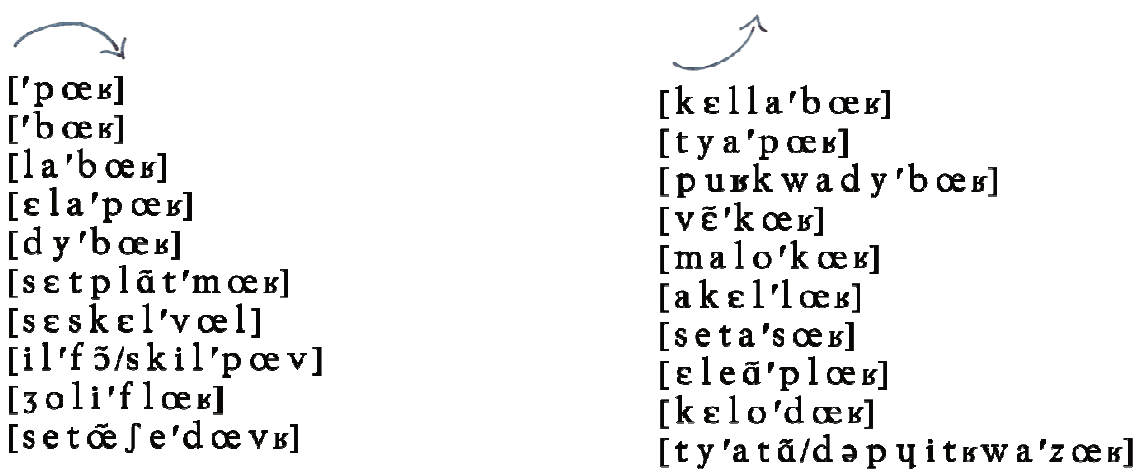

- Tendre le /o/

Dans le but de tendre le /o/, nous proposerions de placer cette voyelle à la fin de courbe intonative montante et l'accompagner des consonnes [p, t, f, s]. Encore une fois, jouer sur le rythme et le débit des énoncés à produire, veiller en même temps à différencier les deux timbres /o/ et /\%/. Ensuite, passer progressivement à un cadre moins favorable.

\begin{tabular}{|c|}
\hline 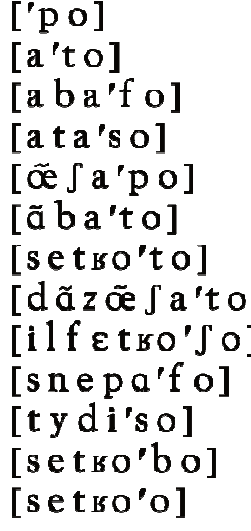 \\
\hline
\end{tabular}

\section{2. ÉTUDE DE CAS : LOCUTRICE 2 (B2-C1 CECRL)}

Au moment de l'étude (2015/2016) : cette locutrice apprend le français depuis quatre ans. Elle a commencé au lycée à Varsovie et elle continue de perfectionner le français en France depuis 7 mois, car elle fait ses études à l'Université de Strasbourg. Elle évalue son niveau de français à B2-C1. Le polonais est sa langue maternelle, elle parle également anglais (B1-B2). 


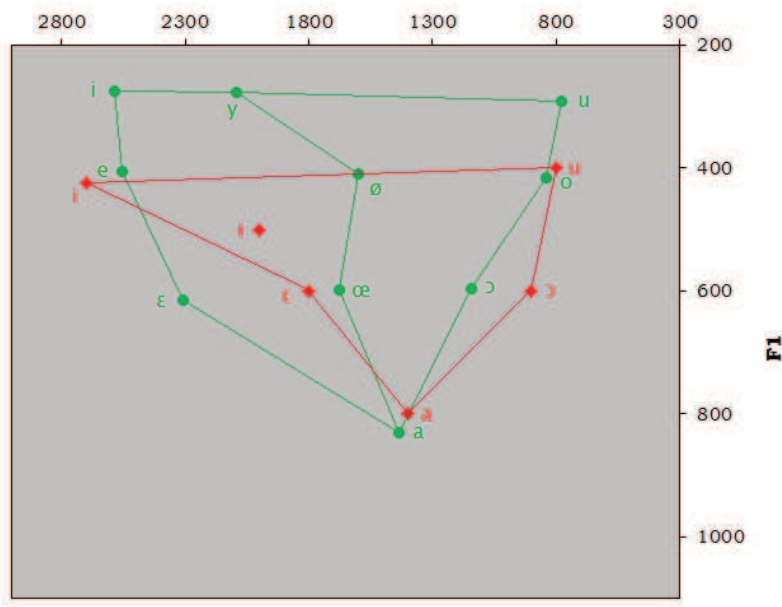

F2

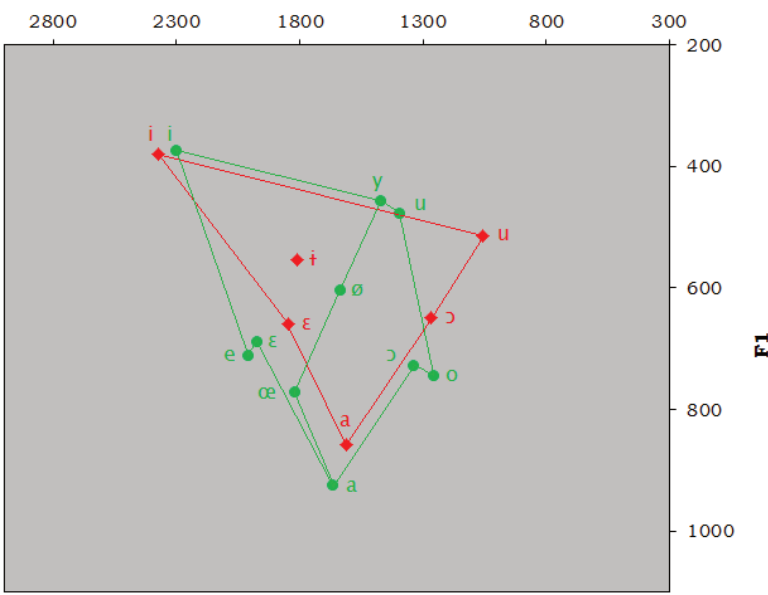

Figure 2 - Locutrice 2 : Superposition des espaces vocaliques des deux langues - valeurs formantiques $(\mathrm{Hz})$ de référence (à gauche), valeurs moyennes formantiques $(\mathrm{Hz})$ de la locutrice 2 (à droite)

Remédiation proposée à partir du diagnostic effectué (Hamm, Dańko, 2017, voir figure 2 pour la synthèse).

En ce qui concerne la locutrice 2 :

- la remédiation envisagée viserait la distinction des voyelles $/ \mathrm{y} /$ et $/ \mathrm{u} / \mathrm{en}$ insistant sur le caractère clair du /y/ et le caractère sombre du /u/ ;

- il serait conseillé de renforcer le caractère tendu du /e/ ;

- tendre le /o/ ;

- assombrir le /œ/. 
Méthodologie de remédiation

- Distinguer les voyelles /y/ et /u/ (voir supra)

- Renforcer le caractère tendu du /e/ (voir supra)

- Tendre le /o/ (voir supra)

- Assombrir le /œ/ (voir supra)

\section{3. ÉTUDE DE CAS : LOCUTRICE 3 (C1 CECRL)}

Au moment de l'étude (2015/2016) : cette locutrice apprend le français depuis 9 ans (avec une pause de trois ans), elle a commencé au lycée en Pologne. Elle étudie à l'Université d'Innsbruck, mais elle habite en Italie. Elle évalue son niveau de

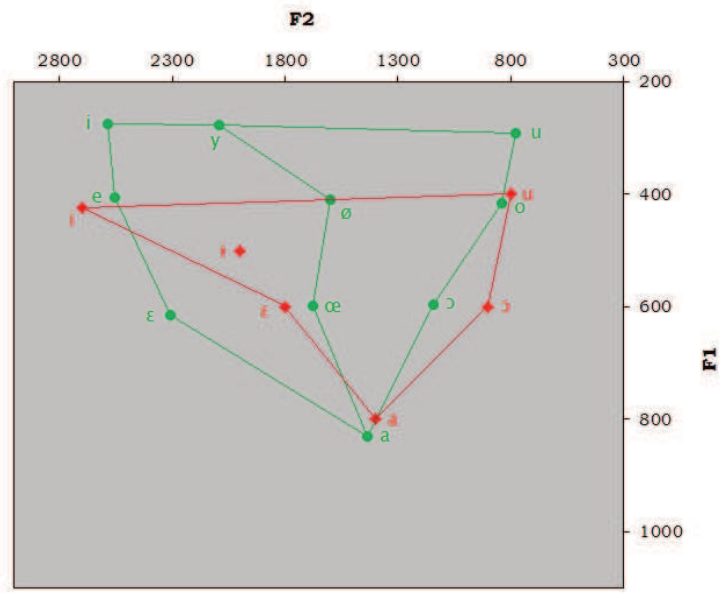

F2

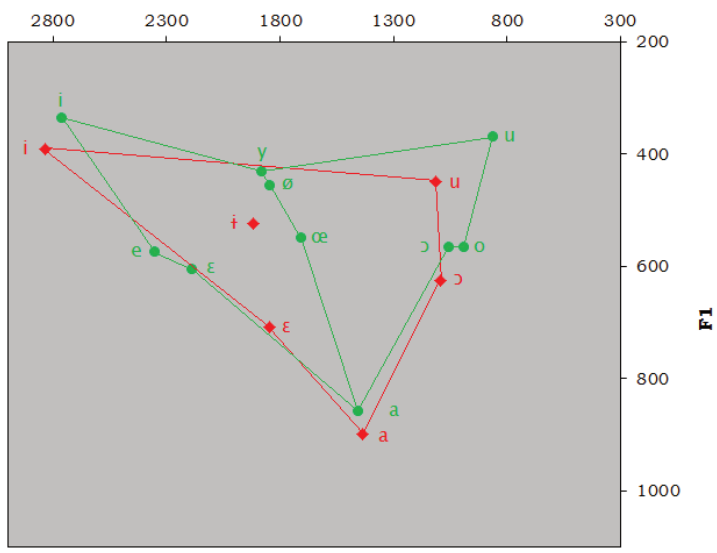

Figure 3 - Locutrice 3 : Superposition des espaces vocaliques des deux langues - valeurs formantiques $(\mathrm{Hz})$ de référence (à gauche), valeurs moyennes formantiques $(\mathrm{Hz})$ de la locutrice 3 (à droite) 
français à C1. Elle parle aussi italien (niveau C2), allemand (niveau C1), anglais (niveau $\mathrm{C} 1$ ), et le polonais est sa langue maternelle.

Remédiation proposée à partir du diagnostic effectué (Hamm, Dańko, 2017, voir figure 3 pour la synthèse).

En ce qui concerne la locutrice 3, nous proposerions les procédés de correction suivants :

- tendre le $/ \mathrm{y} /$;

- tendre légèrement le /e/ (sans trop de répétitions successives) ;

- tendre légèrement le /o/ (sans trop insister);

- relâcher le /E/;

- relâcher le $/ \mathrm{o} /$.

Méthodologie de remédiation

- Tendre le /y/

Pour tendre le /y/, nous insisterons sur le rythme et l'accentuation (nous encouragerons à parler plus vite), en incluant un geste (comme c'était le cas pour tendre le /e/). Nous placerions la voyelle en fin de courbe intonative montante et nous nous servirions de l'entourage consonantique facilitant $[\mathrm{p} t \mathrm{f} s]$. Comme prévu, nous changerions ensuite de contexte pour passer au cadre défavorable.

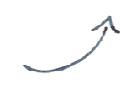

[a'p y ]

[a't y]

[a'f y]

[a'k y]

[py't y]

[ty'f y]

[f y'k y]

[laky l't ye ]

[lali teв a't у в]

[ilf $\varepsilon$ deze'tyd]

[kelavã't y s]

[ã tã't y]

[' $\varepsilon l / \varepsilon \ln$ a pa'p y ]

[setu'f y]

[ y n b on'k y ]

[keskiljaom ə'n y]

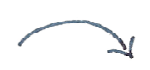

[d ə lo'p у в ]

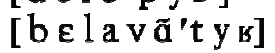

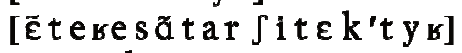

['n $\tilde{s} / \varepsilon \ln$ a pa'p y]

[k omdabi't yd]

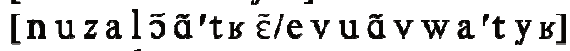

[ynzoli'3 y p]

[3 $\mathrm{n} \varepsilon \mathrm{m}$ 'pa/s $\mathrm{s}$ tkwa'f y $\mathrm{k}$ ]

[pa ' $^{\prime} \mathrm{d} \tilde{\mathrm{s}} / 3$ ว nle $\mathrm{pa} \mathrm{a}^{\prime} \mathrm{v}$ ] ]

[swajelebjẽva'n y]

[थ̈kaf es a 's y kr ]

- Tendre légèrement le /e/

Pour tendre légèrement le /e/, quelques occurrences suffisent pour cette locutrice. Nous conseillerons de privilégier le rythme et donc le débit plus rapide, de placer 
la voyelle en fin de courbe intonative ascendante en incluant l'entourage consonantique facilitant (voir supra).

- Tendre légèrement le /o/

Pour tendre le /o /, voir les procédés proposés supra.

- Relâcher le /E/

Pour relâcher cette voyelle, nous la placerions en fin de courbe intonative descendante, dans l'entourage consonantique [ $\mathrm{b} \mathrm{m} \vee \mathrm{n}$ ], et nous demanderions de parler lentement pour créer des conditions favorables à l'acquisition, ensuite nous changerions de contexte.
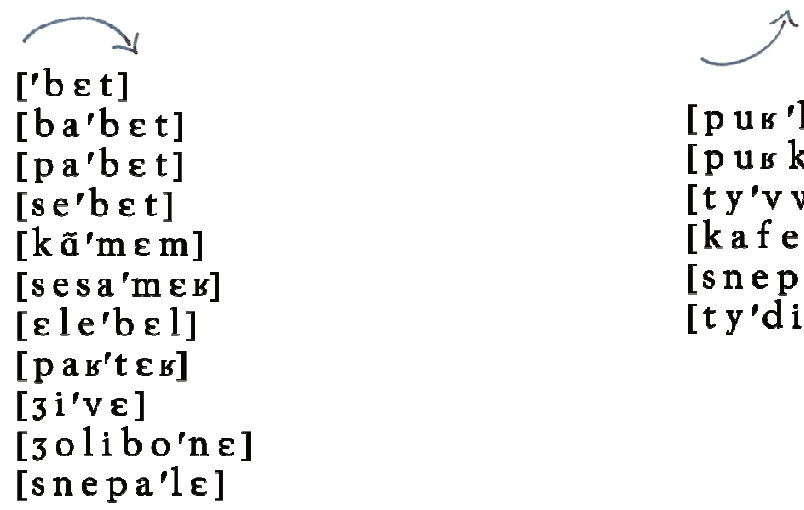

[puк'kwa/ilsi'mel]

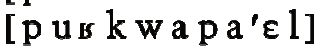

[t y'vwa/s $\varepsilon$ ti $\left.\tilde{s}^{\prime} \mathrm{d} \varepsilon \mathrm{l}\right]$

[kaf e o'l $\varepsilon$ ]

[snepa'le]

[ty'di/siltø'ple]

$\left[\operatorname{pas}^{\prime} t \varepsilon \varepsilon\right]$

[3i've]

[3olibo'n $\varepsilon$ ]

[snepa'le]

- Relâcher le /o/

Pour relâcher le / $/$, de la même manière, nous proposerions de s'exercer à l'aide de mots phonétiques où la voyelle se trouverait en fin de courbe intonative descendante, dans l'entourage consonantique [ $\mathrm{b} \mathrm{m}$ v n], en parlant lentement, pour ensuite changer de contexte.

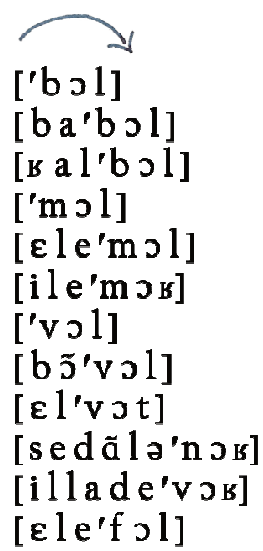

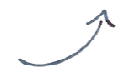

[y n'tas/u án'bol]

[setala'mod]

[kelf a s ã'd ol]

[par a'sol]

[puв kwa'pa/o'sol]

[nes'tos]

[ila'tó ] 


\section{4. ÉTUDE DE CAS : LOCUTRICE 4 (C2 CECRL)}

Au moment de l'étude (2015/2016) : cette locutrice habite depuis six ans à Strasbourg et étudie à l'Université de Strasbourg, c'est pour cela que son niveau de français est très avancé $(\mathrm{C} 2)$. Elle a commencé à apprendre le français au lycée en Pologne. Elle parle aussi anglais (B2) et allemand (B2), le polonais est sa langue maternelle.

Remédiation proposée à partir du diagnostic effectué (Hamm, Dańko, 2017, voir figure 4 pour la synthèse).
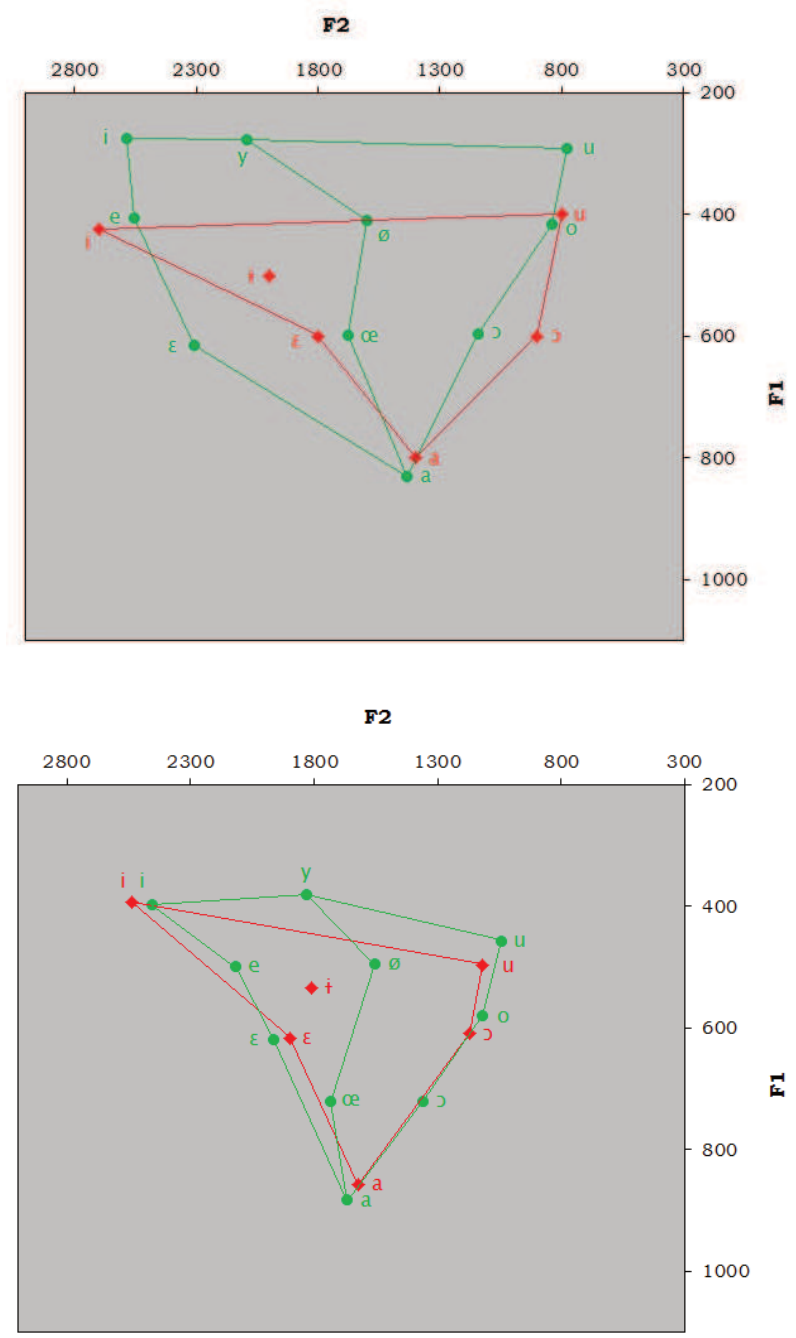

Figure 4 - Locutrice 4 : Superposition des espaces vocaliques des deux langues - valeurs formantiques (Hz) de référence (à gauche), valeurs moyennes formantiques $(\mathrm{Hz})$ de la locutrice 4 (à droite) 
En ce qui concerne la dernière locutrice, nous proposerions les procédés de correction suivants :

- tendre un peu le $/ \mathrm{o} /$

- assombrir le /œ/ ;

- tendre le /œ/ ;

- tendre un peu le $/ \mathrm{s}$.

Méthodologie de remédiation

- Tendre un peu le /o/ (voir supra, sans trop insister)

- Assombrir le /œ/ (voir supra)

- Tendre le /œ/

Pour tendre cette voyelle, nous insisterions sur le rythme et donc un débit plus rapide, l'intonation ascendante (nous placerions la voyelle à la fin de courbe intonative montante) et nous proposerions de la faire précéder des consonnes [p t f s]. Progressivement, nous passerions au cadre moins favorable.

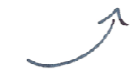

['p $\propto \mathrm{s}]$

[t y a'p $\propto$ в]

[as $\varepsilon$ 't $œ$ r]

[purkwa'pa/a ч i'tœr]

[seta's $œ$ r]

[movezo'dœr]

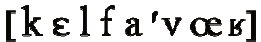

['y $\mathrm{n} / \mathrm{ud} \boldsymbol{d}^{\prime} \mathrm{f} l \mathrm{l}_{\text {в }]}$

[ty a malo'k ке

[puskwa $\varepsilon$ le ã'plœ

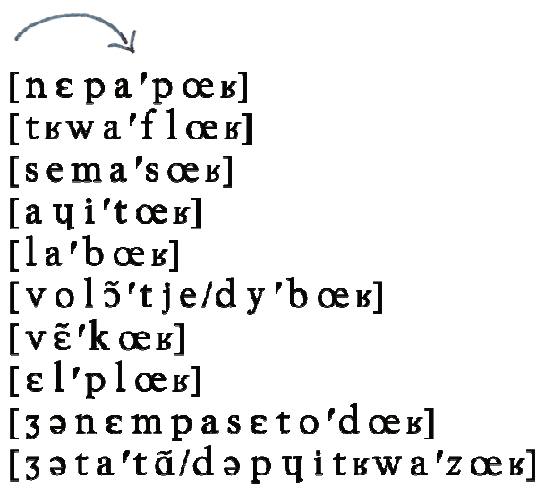

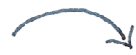

['wi/sem $\tilde{\mathbf{s}}$ 'p $\mathrm{st}]$

['wi/n $\varepsilon s^{\prime} t$ ว

[se's у г/i la't วк ]

['ñ/pao's ol]

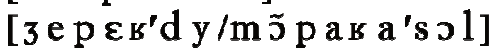

[sneplyal a'mod]

[ $\varepsilon \mathrm{lad}$ is pa'r y/tamas'm $\supset \mathrm{t}$ ]

dlak $\left.\tilde{J}^{\prime} \mathrm{p} \supset \mathrm{t}\right]$

[kel'vot]

[ynbel'not]

[tya'vy/lamas'mot] 
- Tendre un peu le /o/

Pour tendre le /o/, selon les mêmes procédés, nous privilégions toujours le travail sur le rythme, l'accentuation et la façon plus rapide de parler. Nous placerions la voyelle en fin de courbe intonative montante et nous proposerions de la faire précéder des consonnes [p t k]. En ce qui concerne cette locutrice, quelques occurrences suffisent, sans trop insister.

\section{EN GUISE DE CONCLUSION}

Dans une perspective innovante, car à partir des deux corpus de données (français et polonais), nous avons réalisé une étude acoustique expérimentale dont les résultats nous ont servi comme point de départ d'une correction phonétique «sur mesure », puisqu'adaptée aux difficultés réelles rencontrées dans l'acquisition du système vocalique français de quatre locutrices polonophones. Chaque fois, nous avons établi un diagnostic précis, suivi d'une proposition de procédés de correction concrets, avec un exemple de remédiation à adopter. Nous avons privilégié l'approche verbo-tonale de correction phonétique, en prenant en considération respectivement deux traits à corriger : problème d'appréciation du degré de tension (qui correspond aux valeurs du premier formant, F1 de la voyelle en question); problème d'appréciation du degré d'acuité (qui correspond aux valeurs du deuxième formant, F2 de la voyelle en question).

Nous n'avons pas pris en considération le F3 et un éventuel travail sur la labialité, pourtant la prise des mesures du troisième formant (F3) pourrait devenir révélatrice d'autres phénomènes à encadrer, dans une autre perspective que la verbotonale, notamment l'articulatoire, ou encore Silent Way. Quant à la réussite de la remédiation à long terme (pour parler d'une acquisition), celle-ci dépendrait encore d'autres éléments en jeu, à part l'aspect acoustique, il y aurait encore le facteur psychologique (émotionnel, motivationnel), physiologique, interpersonnel ou encore institutionnel. Seule une étude acoustique à partir d'un corpus longitudinal pourrait valider ces acquis ; une étude longitudinale est d'ailleurs actuellement en cours et porte sur l'acquisition du /y/. 


\section{BIBLIOGRAPHIE}

Best, C. (1994). The Emergence of Native-Language Phonological Influences in Infants: A Perceptual Assimilation Model. In J. Goodman, H. Nusbaum H.C. (réd.), The Development of Speech Perception: The Transition from Speech Sounds to Spoken Words (pp. 167-224). Cambridge : Mass MIT Press.

Billerey, B. (2004). La perception des phonèmes français chez les apprenants polonais. Élaboration d'un outil pédagogique en FLE dans l'optique verbo-tonale de correction phonétique. Lille: ANRT.

Billières, M. (2005). Les pratiques du verbo-tonal. Retour aux sources. In M. Berré (réd.), Linguistique de la parole et apprentissage des langues. Questions autour de la méthode verbo-tonale de P. Guberina (pp. 67-87). Mons : CIPA.

Blanchet, Ph. (2016). Discrimination : combattre la glottophobie. Paris : Textuel.

Boersma, P., Weenink, D. (2010). Praat: Doing phonetics by computer (version 5.1.29). Available from http://www.praat.org/ [Computer program].

Callamand, M. (1981). Méthodologie de l'enseignement de la prononciation. Organisation de la matière phonique du français et correction phonétique. Paris : CLE International.

Dańko, M., Sauvage, J., Hirsch, F. (2015). La perception phonémique en français des apprenants polonophones. L'Information grammaticale, 146, 34-40.

Dańko, M., Sauvage, J., Hirsch, F. (sous presse). La perception phonémique en français des apprenants polonophones (le cas des voyelles antérieures de moyenne aperture). Neophilologica.

Detey, S., Kawaguchi, Y. (2008). Interphonologie du Français Contemporain (IPFC) : récolte des données et apprenants japonais. Communication aux Journées PFC : Phonologie du français contemporain : variation, interfaces, cognition, Paris, France, 11-13 décembre 2008. URL : http://cblle. tufs.ac.jp/ipfc/

Flege, J. E. (1995). Second language speech learning: Theory, findings and problems. In W. Strange (réd.), Speech Perception and Linguistic Experience: Theoretical and Methodological Issues in Cross-Language Speech Research (pp. 233-277). Baltimore : York Press.

Gendrot, C., Adda-Decker, M. (2006). Analyses formantiques automatiques en français : périphéralité des voyelles orales en fonction de la position prosodique. In Actes des XXVI journées d'études sur la parole, Dinard, France, juin 2006 (pp. 407-410).

Georgeton, L., Paillereau, N., Landron, S., Gao, J., Kamiyama, T. (2012). Analyse formantique des voyelles orales du français en contexte isolé : à la recherche d'une référence pour les apprenants de FLE. In Actes de la conférence conjointe JEP-TALN-RECITAL 1 (pp. 145-152). Grenoble.

Guberina, P. (1971). Introduction à la méthode verbo-tonale de correction phonétique. Paris : Didier.

Hamm, D., Dańko, M. (2017). Perception et traque de «l'accent étranger » : la production des voyelles orales en FLE par des polonophones. Écho des études romanes, XIII(1), 101-122.

Intravaia, P. (2000). Formation des professeurs de langue en phonétique corrective. Le système verbotonal. Paris : Didier Érudition.

Kamiyama, T., Vaissière, J. (2017). La prononciation des apprenants de FLE et la phonétique expérimentale. In S. Detey, I. Racine, Y. Kawaguchi, J. Eychenne (réd.), La prononciation du français dans le monde - Du natif à l'apprenant (pp. 239-245). Paris : CLE International.

Kuhl, P. (1991). Human adults and human infants show a "perceptual magnet effect" for the prototypes of speech categories, monkeys do not. Perception and Psychophysics, 50(2), 93-107.

Landron, S., Paillereau, N., Nawafleh, A., Exare, Ch., Hirofumi, A., Gao, J. (2010). Le corpus PhoDiFLE : un corpus commun de français langue étrangère pour une étude phonétique des productions de locuteurs de langues maternelles plurielles. Cahiers de praxématique, 54, 73-86. 
Polivanov, E. (1931). La perception des sons d'une langue étrangère. Travaux du Cercle de Linguistique de Prague, 4, 79-96.

Racine, I., Detey, S., Zay, F., Kawaguchi, Y. (2012). Des atouts d'un corpus multitâches pour l'étude de la phonologie en L2 : l'exemple du projet «Interphonologie du Français Contemporain (IPFC)». In A. Kamber, C. Skupiens (réd.), Recherches récentes en FLE (pp. 1-19). Berne : Peter Lang.

Troubetzkoy, N.S. (1949). Principes de phonologie. Paris : Klincksieck.

Wierzchowska, B. (1965). Wymowa polska. Warszawa : Państwowe Zakłady Wydawnictw Szkolnych. Wierzchowska, B. (1980). Fonetyka i fonologia języka polskiego. Wrocław : Ossolineum. 